Revue d'histoire de l'Amérique française

ZAS REVUE D.HISTOIRE DE L'AMÉRIQUE FRANÇAISE

\title{
Propriété foncière et société à Montréal : une hypothèse
}

\section{Paul-André Linteau et Jean-Claude Robert}

Volume 28, numéro 1, juin 1974

URI : https://id.erudit.org/iderudit/303328ar

DOI : https://doi.org/10.7202/303328ar

Aller au sommaire du numéro

Éditeur(s)

Institut d'histoire de l'Amérique française

ISSN

0035-2357 (imprimé)

1492-1383 (numérique)

Découvrir la revue

Citer cet article

Linteau, P.-A. \& Robert, J.-C. (1974). Propriété foncière et société à Montréal : une hypothèse. Revue d'histoire de l'Amérique française, 28(1), 45-65.

https://doi.org/10.7202/303328ar d'utilisation que vous pouvez consulter en ligne.

https://apropos.erudit.org/fr/usagers/politique-dutilisation/ 


\title{
PROPRIETEE FONCIÊRE ET SOCIÉTE À MONTREAL : UNE HYPOTHĖSE *
}

\author{
Paul-André Linteau \\ Département d'histoire \\ Université du Québec à Montréal
}

JeAN-Claude Robert

Groupe de recherche sur la société montréalaise au $19^{e}$ siècle

Université du Québec à Montréal

La propriété foncière urbaine est certes un domaine passablement négligé de l'historiographie québécoise. L'intérêt des historiens s'est surtout porté sur la partie du capital consacrée par la bourgeoisie aux activités commerciales ou manufacturières; on a eu tendance à négliger la part du capital utilisée à l'acquisition du sol urbain et l'intérêt que mettait la bourgeoisie à faire fructifier ces investissements fonciers. ${ }^{1}$

Cette situation peut s'expliquer par les difficultés que présentent l'étude de la propriété foncière et la mise en rapport de celle-ci à la structure sociale. La recherche en ce domaine requiert de longs et minutieux travaux et la mise en œuvre de moyens considérables. L'objectif du présent texte est plus modeste. Il vise à formuler une première hypothèse à propos de l'inser-

* Cette étude s'inscrit dans le cadre des travaux du Groupe de recherche sur la société montréalaise au $19 \mathrm{e}$ siècle (GRSM) dirigé par Jean-Paul Bernard, Michel Grenon et Paul-André Linteau, du département d'histoire de l'Université du Québec à Montréal. Le présent travail a été rendu possible grâce à une subvention du Ministère de l'Education du Québec (Programme de formation de chercheurs et d'action concertée). Nous remercions nos collègues Jean-Paul Bernard, Louise Dechêne et Normand Séguin, qui nous ont fait de précieux commentaires.

1 Parmi les rares études sur le sujet il faut mentionner l'article de Louise Dechêne, "La croissance de Montréal au XVIIIe siècle", RHAF, 27, 2 (septembre 1973) : 163-179 et la thèse de doctorat du même auteur, Habitants et marchands de Montréal au XVIIe siècle, Thèse de doctorat d'Etat (Paris, 1973). Au plan méthodologique on ne saurait passer sous silence le travail accompli présentement à l'Université du Québec à Chicoutimi par le Groupe de recherche sur la propriété foncière au Saguenay; voir Pierre Houde et Normand Séguin, Rapport d'activité du Groupe de recherche sur la propriété foncière au Saguenay pour l'année de subvention mai 1973 à mai 1974 (novembre 1973), 53 p. (Miméo).

RHAF, vol. 28, no 1 (juin 1974) 
tion du capital foncier dans l'activité économique et surtout de la place qu'y occupe la bourgeoisie canadienne-française. Pour y arriver nous nous appuyons sur les indices que nous fournissent certaines recherches en cours et plus spécifiquement sur les résultats que livre une première étude, encore assez générale, de la propriété foncière à Montréal en 1825. Ce texte n'est donc pas un exposé de résultats définitifs. Il ne prétend pas non plus formuler une problématique bien articulée, mais simplement avancer une hypothèse qui devra être ensuite vérifiée et affinée, au besoin, par une application à des cas concrets, y compris celui de Montréal. C'est, en réalité, un point de départ pour la recherche.

En première partie nous décrirons brièvement les sources les plus importantes pour l'étude du capital foncier. Nous nous pencherons ensuite sur la situation de la propriété foncière montréalaise en 1825 , année pour laquelle nous possédons des données assez précises compilées par Jacques Viger, le principal fonctionnaire de la municipalité, à l'époque. ${ }^{2}$ Nous tenterons enfin de dégager les éléments d'une hypothèse plus globale concernant le rôle de la propriété foncière à Montréal et l'action de la bourgeoisie canadienne-française dans ce secteur.

Avant d'aborder le sujet proprement dit il faut évoquer le cadre juridique dans lequel il s'insère.

\section{Le cadre juridique}

Le cadre juridique de la propriété foncière à Montréal est formé par le régime seigneurial, avec en arrière-plan, la coutume de Paris qui en régit l'application. Cette situation dure jusqu'à la deuxième moitié du $19 \mathrm{e}$ siècle alors que l'abolition du régime et la confection du Code civil crêent un tout nouveau cadre.

La cité de Montréal est englobée dans la seigneurie du même nom, propriété depuis 1663 du Séminaire de Saint-Sulpice de Paris et, depuis 1764, du Séminaire de Saint-Sulpice de Montréal. ${ }^{3}$

2 La documentation utilisée provient du fonds Viger-Verreau, déposé aux Archives du Séminaire de Québec. Nous tenons à remercier le personnel des Archives et en particulier son directeur, M. l'abbé Honorius Provost, pour toute l'aide qu'il nous a apportée.

3 Voir G.-E. Baillargeon, La survivance du régime seigneurial d̀ Montréal (Montréal, Le Cercle du Livre de France, 1968). 
Rappelons ici quelques notions de droit seigneurial: La terre fait l'objet d'au moins deux types de propriété; le premier appelé domaine utile correspond à la propriété du censitaire qui jouit de son bien, et le second, connu sous le nom de domaine direct, ou tout simplement la directe, fonde les droits seigneuriaux. Très tôt cependant, dès le XIVe siècle, le censitaire est reconnu comme propriétaire, au sens plus moderne du terme. ${ }^{4}$ La directe n'apporte au seigneur que le bénéfice des droits réels fixes (cens et rentes) et casuels (les plus connus - et surtout les plus rentables - étant les lods et ventes).$^{5}$ Donc à l'intérieur de la cité de Montréal, les censitaires peuvent disposer de leur bien comme bon leur semble à condition de payer les droits au seigneur, spécialement ceux de mutation. Il persiste certains privilèges seigneuriaux (les banalités, etc.), mais ils n'empêchent pas les ventes. Les droits de mutation étant proportionnels à la valeur des biens-fonds ils apparaissent très tôt intolérables à la bourgeoisie montréalaise, de même que certaines banalités. Depuis qu'en 1816 les seigneurs avaient été déboutés d'une poursuite contre un certain Fleming, ils n'osaient d'ailleurs pas trop poursuivre pour les recouvrements de lods et ventes et même de cens et rentes.

Toutefois, cette question de la précarité des droits seigneuriaux des Sulpiciens n'a pas de rapport avec l'administration plus proprement municipale. La principale marque du régime seigneurial sur le paysage montréalais se situe au plan du parcellaire; il faudrait déterminer de quelle façon les lots étaient découpés, et aussi comparer les pratiques des possesseurs des arrière-fiefs en ce domaine. Nous croyons que ces derniers ont, en général, loti leurs propriétés en terrains plus petits et cela plus tôt qu'ailleurs. Mais tout cet aspect de la création du parcellaire urbain est trop complexe pour être plus qu'évoqué ici.

\section{Les sources}

Les sources permettant d'étudier la propriété foncière sont nombreuses mais leur utilisation s'avère souvent difficile. Les

\footnotetext{
4 Jean-Philippe Lévy, Histoire de la propriété (Paris, P.U.F., 1972), 46.

5 Sur la question du droit et de l'installation du régime à Montréal voir: Louise Dechêne, "L'évolution du régime seigneurial au Canada", Recherches Sociographiques, XIF, 2 (mai-août 1971): 143-183 et Yves F'. Zoltvany, "Esquisse de la Coutume de Paris", RHAF, 25, 3 (décembre 1971) : 372-373.
} 
greffes de notaires sont bien connus. Ils recèlent un grand nombre de contrats et d'actes divers permettant de connaître les mutations de propriétés, la valeur marchande de celles-ci, l'utilisation du sol, les constructions qui sont érigées, etc. Très précieux au niveau micro-historique, pour l'étude de tel ou tel lot, des possessions de tel propriétaire, ou même pour la connaissance de l'état du marché foncier à un moment précis, ils permettent difficilement de reconstituer un tableau d'ensemble de la propriété foncière à l'échelle d'une ville. Il faut plutôt les considérer comme des sources complémentaires venant étoffer les données de nature sérielle obtenues par ailleurs.

Plus faciles d'accès sont les archives des bureaux de conservation des titres. ${ }^{6}$ A Montréal, celles-ci n'apparaissent qu'en 1842 avant même la confection du cadastre qui inscrit officiellement le bornage et la superficie des terres. Antérieurement seul existait le terrier établi pour les Sulpiciens, seigneurs de l'île de Montréal. Les annotations comprises dans ce document d'envergure s'étendent des débuts de Montréal jusqu'aux années 1860. Les indications contenues dans le terrier sont souvent vagues, les dimensions et le bornage imprécis et la quantité d'informations plutôt mince, si on compare cette source avec les données des bureaux d'enregistrement de la période postérieure. Là encore nous sommes en présence d'une source qui se prête bien à des études micro-historiques mais qui rend plus difficile l'examen, en coupe instantanée, de l'état de la propriété foncière dans l'ensemble de la ville.

Dans ces circonstances il devient essentiel de recourir aux sources municipales: les rôles d'évaluation, confectionnés annuellement à Montréal depuis 1847, et leurs ancêtres les rôles de cotisation. Ces registres naissent de la nécessité de répartir sur l'ensemble des propriétaires et au prorata de la valeur de leurs terrains, le coût des travaux de voirie. Mention en est faite pour la première fois dans l'“Acte pour faire, réparer et changer les chemins et ponts dans cette Province et pour d'autres effets". ${ }^{7}$ Cette loi forme d'ailleurs la base des administrations paroissiales et municipales qui régiront le Bas-Canada jusqu'à la structuration d'un véritable système municipal en 1855 .

6 On trouvera une description de ce type de sources dans Gérard Bouchard et Normand Séguin, "Pour une histoire de l'occupation du sol et de la propriété foncière au Saguenay", Protée, 1, 3 (décembre 1971): 33-39 et Gilles Durand, "Sources manuscrites pour l'histoire de la vallée du Haut-Saguenay et du Lac Saint-Jean", Protée, 2, 1 (avril 1972) : 103-112.

7 Statuts du Bas Canada, 36 George III (1796), chap. 9. 
Voyons ce que la loi de 1796 prévoit au chapitre de la cotisation. Distinguons dès maintenant cotisation et corvée. Cette dernière obligation frappe tous les hommes de 18 à 60 ans. Seuls en sont exemptés les apprentis et les étudiants, les chargés de famille de "jeunes enfants", ceux qui furent victimes d'une maladie ou d'une infirmité dans l'année et les militaires à l'exception curieusement, des officiers d'état-major. Il est possible de remplacer la corvée par une taxe fixe; à ce moment on paie la "composition" pour le nombre de jours de corvée décrétés par l'inspecteur des chemins ou ses assistants. La cotisation, elle, frappe uniquement la propriété foncière. Annuellement, cinq cotiseurs, ou pour reprendre le texte de la loi, "cinq Domiciliés honnêtes", entreprennent d'évaluer le revenu annuel de chacune des propriétés se trouvant à l'intérieur du périmètre de la cité. Ils sont nommés par les juges responsables de l'administration. Cette évaluation peut faire l'objet d'un recours légal, à défaut duquel elle est réputée juste et la cotisation, fixée en 1796 à 4 deniers par livre (cours d'Halifax), est levée et perçue par les sous-inspecteurs des chemins, dirigés par l'inspecteur.

Seules quelques catégories de propriétaires échappent à la cotisation: les propriétés d'une valeur inférieure à $£ 5$., celles des communautés religieuses et les terrains sis à l'extérieur des fortifications mais à l'intérieur des limites de la cité, et qui servent de prairie, de pâturage ou à la culture du grain. Notons que la loi ne prévoit pas d'exemption pour les églises ou les propriétés gouvernementales.

Trois ans plus tard, en 1799, on modifie cette loi. ${ }^{8}$ Pour la partie qui nous intéresse notons que le taux de la cotisation peut être porté de 4 à 6 deniers par livre et que les terrains servant de prairie, de pâturage ou à la culture du grain situés hors des limites des fortifications, perdent leur exemption.

Enfin, d'autres taxes existent à Montréal; elles frappent les propriétaires de chevaux et de "maisons publiques". La fiscalité municipale est en plein essor car, après 1823, on ajoute la taxe sur les chiens, les voitures, les chevaux encore une fois, les débits de boisson de tous genres et enfin les encanteurs. ${ }^{9}$ Cet essor de la fiscalité explique pourquoi Jacques Viger, dans une lettre à un neveu dans laquelle il demande un tableau des revenus de la ville, termine la liste des diverses taxes par "etc. à mesure qu'il y a de nouveaux genres de revenu".10

8 Ibid., 39 feorge III (1797), chap. 5.

9 Ibid., 3 George IV (1823), chap. 6.

1031 décembre 1825, Jacques Viger à Pre Auger, Saberdache (Bleue), vol. 8: 275-276, Archives du Séminaire de Québec. 
En 1825, le taux de la cotisation est de 6 deniers par livre de revenu annuel évalué, ce qui équivaut à $2.5 \%$. Ce taux et le montant payé en taxe font l'objet d'une inscription dans un registre, appelé livre de cotisation.

Cependant, si les rôles d'évaluation de la cité de Montréal ont été très bien conservés pour la période de 1847 à nos jours il n'en va pas de même pour ceux de la période antérieure. De tels registres ont bel et bien existé avant 1847, sinon de façon continue, au moins pour certaines années. Jacques Viger a confirmé l'existence d'un rôle de cotisation pour 1825, qu'il a luimême consulté, et la disparition subséquente de cette source. ${ }^{11}$

Fort heureusement Viger avait pu, avant cette disparition, établir des statistiques précises sur la propriété foncière et les propriétaires de la cité de Montréal en $1825 . .^{12}$ Si ces compilations, portant sur une seule année, ne rendent pas compte des dynamismes fonciers dans leur dimension temporelle elles permettent une étude approfondie, en coupe instantanée. Il est alors possible de tracer un portrait de la propriété foncière à Montréal en 1825, portrait qui reste toutefois incomplet puisque les renseignements recueillis révèlent seulement le nom et l'origine ethnique du propriétaire, ainsi que le nombre et le revenu de ses biens-fonds, ${ }^{13}$ le tout présenté en fonction des divisions administratives de la cité (ville et faubourgs). Ces données ne fournissent aucune indication sur certains éléments essentiels à une

11 Jacques Viger, Saberdache (Bleue), vol. 6 et 7 , correspondance, passim, Archives du Séminaire de Québec. Les livres de cotisations étaient conservés par les cotiseurs qui n'étaient pas tenus de les déposer au Greffe de la Cour.

12 Ces documents se retrouvent à travers le fonds Viger-Verreau des Archives du Séminaire de Québec. Voici les principales sources utilisées: Recensement de Montréal en 1825, Livre de dépouillement par Jacques Viger (015 A), Recensement de la Ville de Montréal fait par Jacques Viger en 1825 pour Louis-Jos. Papineau (Carton 46, no 9). Documents sur la propriété foncière (Carton 46, no 9). Tablettes statistiques du Comté de Montréal $1825(017)$. Ces dernières ont été brièvement analysées et complétées dans J.-P. Bernard, P.-A. Linteau et J.-C. Robert, "Les tablettes statistiques de Jacques Viger (1825)", Groupe de recherche sur la société montréalaise au 19e siècle, Rapport 1972-1973 (Montréal, Université du Québec à Montréal, 1973), 42 p. (miméo).

13 Tout au long de ce texte l'expression "revenu annuel" désigne l'estimé, établi par les cotiseurs, du montant que peut tirer annuellement un propriétaire de ses biens-fonds. Il ne faut donc pas le confondre avec le revenu réel qui peut être différent et qui ne nous est pas connu. Il est évident que l'estimé des cotiseurs utilisé ici doit être considéré comme un indice permettant des comparaisons et qu'il ne rend pas compte du prix que pourrait valoir un terrain en particulier sur le marché. 
étude exhaustive de la propriété foncière: la superrficie du terrain, le nombre et la qualité des bâtiments qui y"sont érigés:

\section{La propriété}

En 1825 la cité de Montréal ${ }^{14}$ compte 2,698 propriétés cotisées, dont le revenu global annuel est évalué à 88,594 livres. La moyenne du revenu annuel de la propriété s'élève à 33 livres ${ }^{15}$, ce qui semble un chiffre plutôt médiocre.

La répartition du nombre des propriétés entre la ville et les faubourgs suit, d'assez près, celle de la population. La ville compte $23.7 \%$ de la population totale et $22.7 \%$ du nombre des propriétés; les faubourgs hébergent $\mathbf{7 6 . 2} \%$ de la population avec $77.2 \%$ des propriétés. Les chiffres deviennent intéressants lorsque l'on s'interroge sur la valeur moyenne du revenu annuel dans la ville et les faubourgs. Alors que pour l'ensemble, la moyenne du revenu s'établit à 33 livres, le chiffre atteint 82 livres pour la ville et se réduit, au niveau des faubourgs, à un faible 18 livres. A vrai dire, il n'y a dans ce phénomène de la valeur différentielle rien de nouveau, ne serait-ce qu'à cause des matériaux de construction - , la pierre dans la ville et le bois dans les faubourgs - ou à cause de la réglementation urbaine toujours moins vigilante dans les faubourgs que dans les parties centrales des villes. Toutefois, l'ampleur même de cette différence est remarquable puisque les propriétés de la ville vaudraient en moyenne plus du quadruple de la valeur de celles des faubourgs.

14 Trois termes reviennent souvent, pour désigner Montréal, dans les textes de 1825; ce sont la cité de Montréal, la ville de Montréal et les faubourgs. Cité et ville de Montréal occasionnent facilement des confusions; il ne's'agit pas de la même chose. Le premier terme, cité de Montréal, désigne l'unité administrative délimitée et créée par la proclamation de 1792, et englobe la ville et les faubourgs. Le second terme, ville de Montréal, s'applique exclusivement à l'espace circonscrit par le périmètre des anciennes fortifications; c'est la vieille ville du Régime français. Quant aux faubourgs ils se développent à partir du XVIIIe siècle et en 1825 on en compte sept, si l'on assimile la Pointe-à-Callière à un faubourg. Les six autres sont: Sainte-Anne, Saint-Joseph ou des Récollets, Saint-Antoine, Saint-Laurent, Saint-Louis, Sainte-Marie ou Québec. Pour plus de détails, voir P.-A. Linteau et J.-C. Robert, "Les divisions territoriales à Montréal au 19e siècle", Groupe de recherche sur la société montréalaise au $19 \mathrm{e}$ siècle, Rapport 1972-1973 (Montréal, Université du Québec à Montréal, 1973), 32 p. (miméo).

15 En complétant à l'entier le plus rapproché. 
Si nous comparons les faubourgs entre eux, celui qui montre la valeur moyenne la plus faible est le faubourg Québec, avec 16 livres, et la plus élevée le faubourg Sainte-Anne avec 23 livres. La Pointe-à-Callière, qui deviendra plus tard partie intégrante du faubourg Sainte-Anne, doit être traitée à part. En regard des autres parties de la cité, ses caractéristiques semblent, à première vue, aberrantes: comptant $2.7 \%$ de la population totale, elle ne groupe que $0.7 \%$ des propriétés et le revenu moyen s'établit à 90 livres. Ce secteur subit en 1825 les effets de l'ouverture du canal Lachine; on y retrouve une forte proportion d'Irlandais et l'entassement par maison y est prodigieux. En effet, pour l'ensemble de la cité, le nombre d'habitants par maison s'établit à 7.7; pour la Pointe-à-Callière on atteint 18.2 ! Si l'on défalque l'hôpital général des Sœurs Grises (155 personnes) la moyenne ainsi pondérée reste tout de même à 13.7 soit à peu près le double du rapport constaté dans toutes les autres parties de la cité. Haute densité de population et haut revenu foncier, c'est là une situation classique de l'exploitation foncière urbaine au $19 \mathrm{e}$ siècle, en particulier dans les zones peuplées d'immigrants. A l'exception de la Pointe-à-Callière et dans une moindre mesure de Sainte-Anne, les faubourgs ont une forte majorité (plus de $68 \%$ ) de très petites propriétés, c'est-à-dire rapportant moins de 18 livres de revenu annuel. Dans Sainte-Anne ce type de propriété ne représente que $55 \%$ du total, ce qui pourrait s'expliquer par la création relativement récente de ce faubourg, la propriété $\mathrm{y}$ étant moins morcelée.

La figure 1 donne la répartition procentuelle de la valeur des propriétés et permet de voir la structure de la propriété foncière dans la cité. La première colonne représente l'ensemble de la cité $(2,698$ propriétés), la seconde les propriétés de la ville (613) et la troisième celles des faubourgs $(2,085)$. Les moyennes respectives des revenus de la cité (33 livres), des faubourgs (18 livres) et de la ville (82 livres) ont servi à délimiter les quatre classes; ce découpage permet de mieux voir la répartition globale de la propriété.

Nous voyons tout de suite que les valeurs élevées de la propriété dans la ville sont annulées au niveau de l'ensemble par l'étendue de la propriété médiocre. Si la ville compte 613 propriétés dont le revenu évalué s'inscrit entre 6 et 425 livres par année, les faubourgs en comptent 522 qui ne rapportent que 6 livres par année; il y a là de quoi déprimer une moyenne ! D'autre part, la proportion des revenus élevés (au-delà de 100 livres) 


\section{FIGURE 1}

Répartition procentuelle des revenus annuels estimés des propriétaires de la Cité de Montréal en 1825

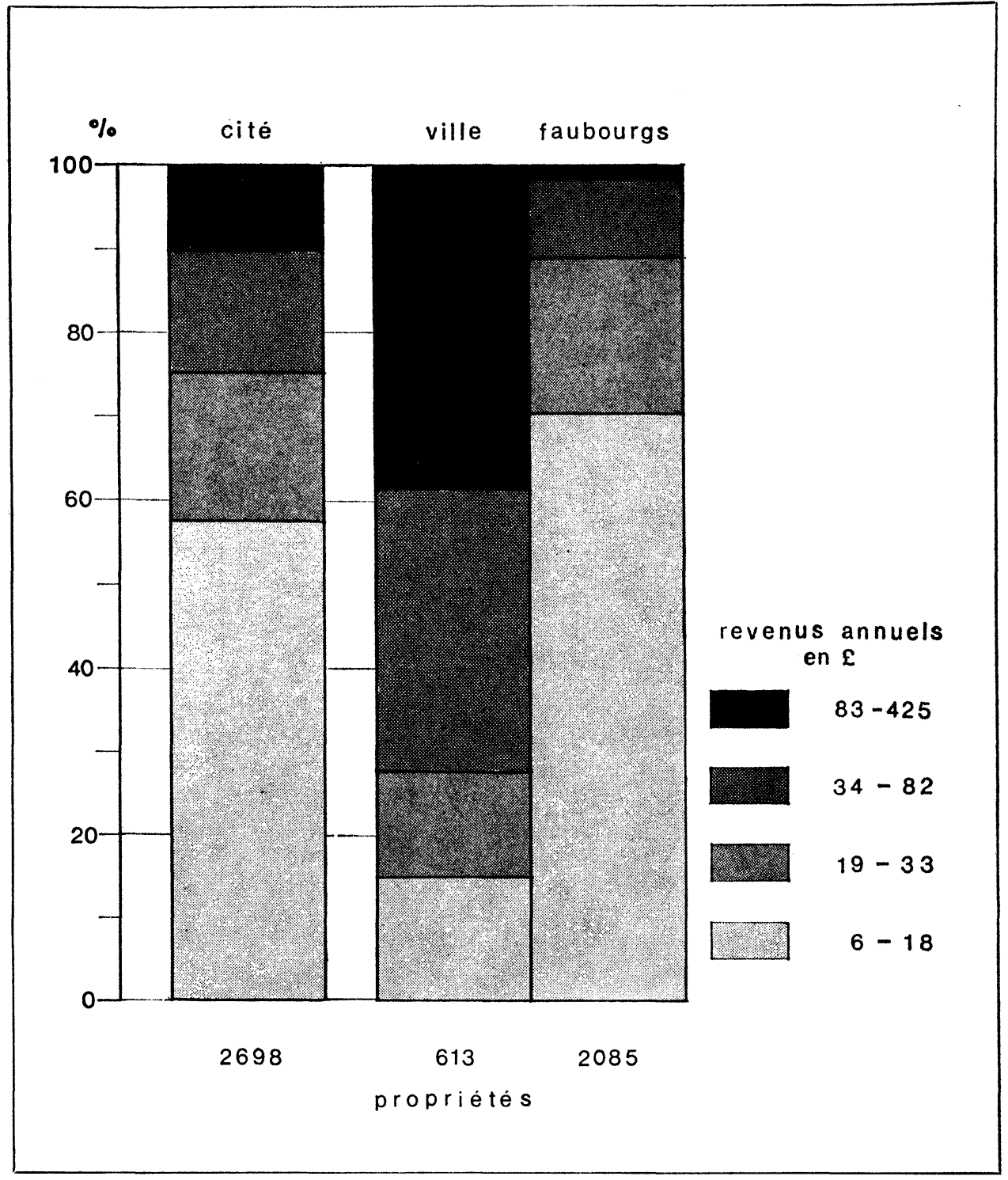


demeure faible: 230 propriétés; formant $8.5 \%$ seulement du total des propriétés, et concentrées à $87 \%$ à l'intérieur de la ville. A regarder ces: colonnes, on pourrait presque se croire en présence de deux villes différentes.

A l'aide des statistiques concernant le matériau de construction des divers bâtiments, nous pouvons poursuivre un peu plus avant cette analyse rapide de la propriété foncière. Le bâtiment est solidaire du fonds de terre dans l'évaluation de la propriété à Montréal; en principe, il devrait y avoir corrélation entre la valeur de la propriété et le matériau; toutefois étant donné le peu de renseignements dont nous disposons, nous ne pourrons aller bien loin: le couple pierre-bois reste un indicateur grossier car il est des maisons de bois de valeur considérable et inversement, on trouve des maisons de pierre de valeur moyenne.

Dans la cité de Montréal, le bois domine avec $64.3 \%$ des maisons recensées alors que $31.8 \%$ sont en pierre. En 1825, la brique fait une timide apparition: dans $3.7 \%$ des maisons elle est utilisée seule ou en combinaison avec le bois.

La répartition des constructions entre la ville et les faubourgs montre l'écrasante présence de la pierre dans les constructions de la ville $(86.5 \%$ des bâtiments) tandis qu'on y relève à peine $6.3 \%$ de constructions de bois. Toutefois, la ville n'a pas le monopole des maisons de pierre puisque $38.3 \%$ d'entre elles se trouvent réparties dans les faubourgs. A l'inverse bien sûr, ces derniers sont massivement en bois (81.2\% des constructions). Remarquons cependant que la variété semble plus grande dans les faubourgs que dans la ville; même si le bois est dominant, il l'est moins que la pierre dans la ville. Nous pouvons esquisser les très grandes lignes de la répartition spatiale de l'activité économique, à l'aide de la recension des ateliers, usines, magasins et comptoirs. Dans la cité, on relève en 1825:161 bâtiments de cette catégorie. On voit encore une fois la prédominance de la ville; $58 \%$ de ceux-ci s'y trouvent, le $42 \%$ restant se ventilant à travers les faubourgs. On en compte partout, mais principalement dans les faubourgs de Saint-Laurent, de Sainte-Anne et de la Pointe-à-Callière.

Voilà donc pour le moment ce que l'on peut tirer d'une analyse rapide de la propriété foncière. Les grandes conclusions de cette analyse sont d'une part l'opposition ville-faubourgs, et ce à tous les niveaux: revenus, qualité des constructions, activité économique; et d'autre part, l'ampleur de la différence de la valeur des propriétés entre les deux parties de la cité. 


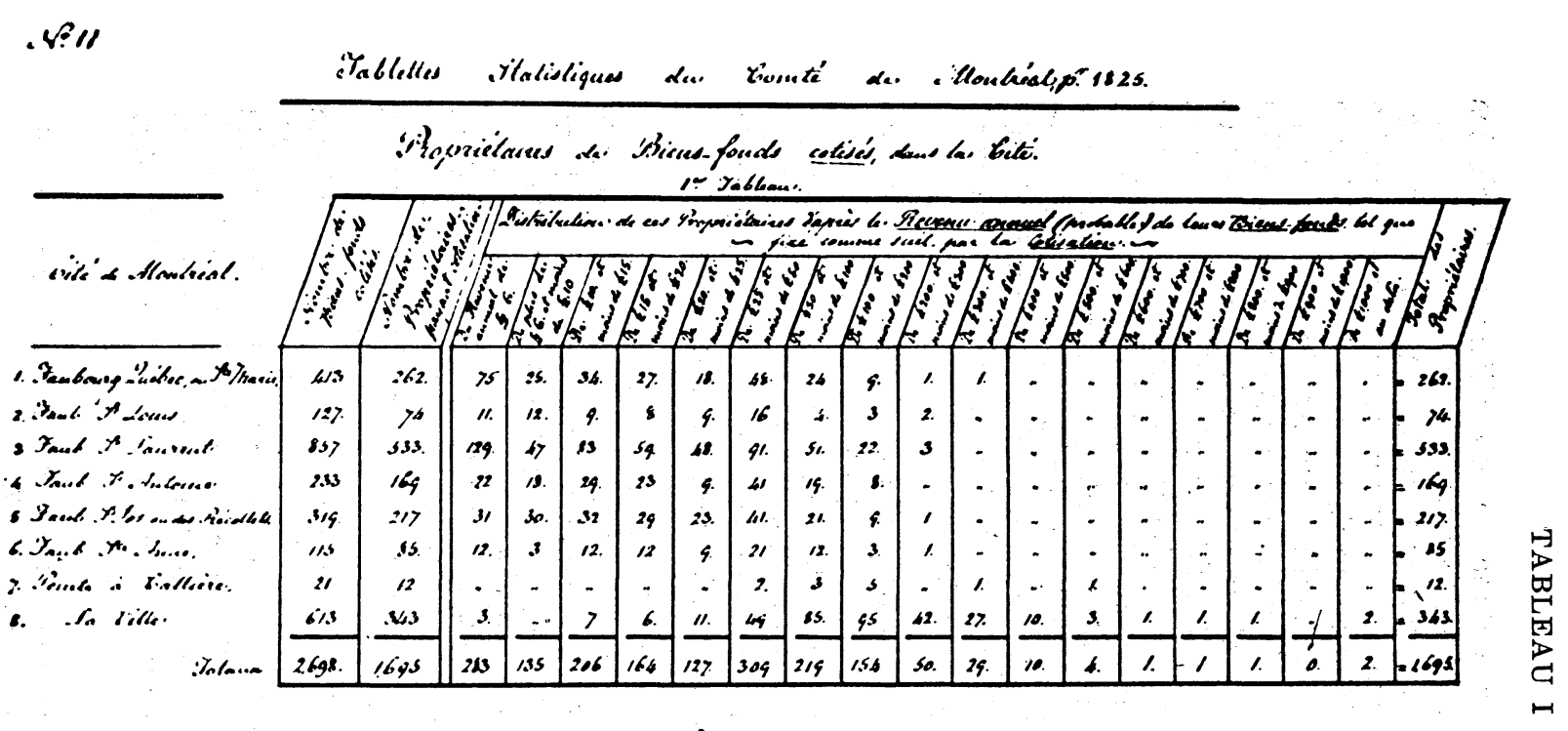

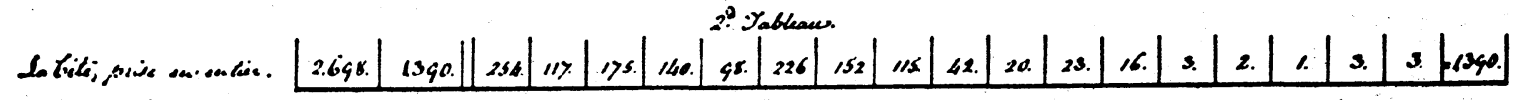

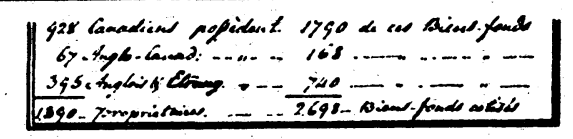

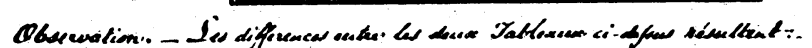

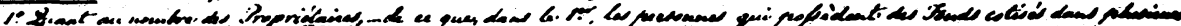

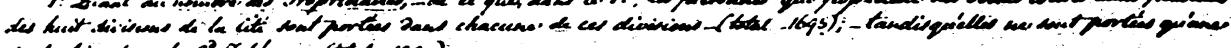

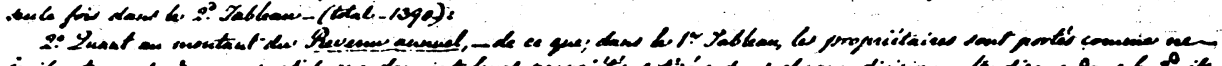

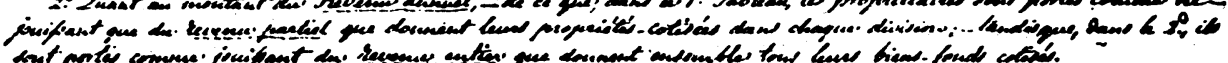




\section{Les propriétaires}

Jacques Viger a consacré l'une de ses célèbres “Tablettes statistiques" aux propriétaires de biens-fonds soumis à la cotisation (tableau 1). Dans un premier temps, il répartit les propriétaires selon des catégories de revenu pour chaque partie de la cité. Eliminant ensuite les comptes doubles, il établit une compilation pour l'ensemble de la cité. On constate que le revenu foncier est très inégalement réparti. Il $\mathrm{y}$ a une masse de petits propriétaires puisque $18.3 \%$ ont un revenu estimé à 6 livres et qu'au total plus de la moitié $(56.4 \%)$ tirent moins de 25 livres par an de leurs biens-fonds. Les catégories de 25 à 50 livres et de 50 à 100 livres rassemblent respectivement 16.2 et $10.9 \%$ des propriétaires. Enfin ceux qui obtiennent un revenu assez substantiel (100 livres et plus), tout en étant minoritaires, n'en représentent pas moins $16.4 \%$ de l'ensemble. Notons toutefois que les très riches propriétaires (500 livres et plus) ne sont qu'au nombre de 28 , soit $2 \%$ du total.

Les évaluateurs ont donc relevé 1,390 propriétaires distincts dans l'ensemble de la cité. Si on considère que celle-ci compte, d'après le recensement de Viger, 4,452 chefs de famille ${ }^{16}$, la mise en rapport de ces deux chiffres indiquerait qu'en 1825 Montréal est déjà nettement une ville de locataires, puisque pas plus de $31 \%$ des chefs de famille y seraient propriétaires.

Jacques Viger a effectué d'intéressants calculs à propos de l'origine ethnique de ces propriétaires. Il a retenu trois grands groupes: les Canadiens (francophones), les Anglo-Canadiens et finalement les Anglais et étrangers. Grâce à ces chiffres il est possible d'établir l'importance relative de chaque groupe. Les Canadiens français qui représentent $54.4 \%$ de la population montréalaise rassemblent les deux tiers des effectifs des propriétaires et les deux tiers des biens-fonds mais n'obtiennent que $52.1 \%$ du revenu évalué, ce qui indique déjà une concentration dans les propriétés à faible revenu. Les Anglo-Canadiens (12.6\% de la population) ne comptent que pour $6.2 \%$ des effectifs des propriétaires et ne détiennent que $4.8 \%$ des biens-fonds dont ils tirent néanmoins $9.6 \%$ du revenu foncier de la cité. Plus forte est la présence des Anglais et autres étrangers (33.0\% de la population) qui avec $27.5 \%$ des propriétaires et $28.4 \%$ des terrains obtiennent $38.3 \%$ du revenu total.

16 "Tablettes statistiques du comté de Montréal, 1825", Archives du Séminaire de Québec, Fonds Viger-Verreau 017. 
TABLEAU 2

Répartition des propriétaires par catégorie de revenu foncier Cité de Montréal 1825

Montant de

l'évaluation du

revenu annuel

(livres)

600 et plus

500-599

$400-499$

300-399

200-299

100-199

$50-99$

$25-49$

20-24

15-19

10-14

6-9

6

TOTAL

\begin{tabular}{rrr}
\multicolumn{3}{c}{ Canadiens français } \\
& $\%$ & $\%$ \\
$\mathrm{n}$ & hor. & vert. \\
& & \\
3 & 60.0 & 0.3 \\
2 & 50.0 & 0.2 \\
3 & 30.0 & 0.3 \\
13 & 44.8 & 1.2 \\
23 & 46.9 & 2.1 \\
77 & 49.7 & 6.9 \\
103 & 47.0 & 9.2 \\
188 & 60.8 & 16.9 \\
80 & 63.0 & 7.2 \\
116 & 70.7 & 10.4 \\
155 & 75.3 & 13.9 \\
117 & 86.7 & 10.5 \\
233 & 82.3 & 20.9 \\
& &
\end{tabular}

\section{Anglo-Canadiens}

$\begin{array}{cc}\% & \% \\ n & \text { hor }\end{array}$

120

0

$1 \quad 10.0$

$3 \quad 10.3$

14.3

9.7

11.4

$4.9 \quad 16.1$

$4.7 \quad 6.5$

$6.1 \quad 10.8$

$1.9 \quad 4.3$

$1.5 \quad 2.2$

$1.4 \quad 4.3$

1,113
10.5
20.9

93

\begin{tabular}{|c|c|c|c|}
\hline \multicolumn{3}{|c|}{ Anglais et étrangers } & \multirow{3}{*}{$\begin{array}{c}\text { Total } \\
n\end{array}$} \\
\hline & $\%$ & $\%$ & \\
\hline $\mathbf{n}$ & nor. & vert. & \\
\hline 1 & 20.0 & 0.2 & $\mathbf{5}$ \\
\hline 2 & 50.0 & 0.4 & 4 \\
\hline 6 & 60.0 & 1.2 & 10 \\
\hline 13 & 44.8 & 2.7 & 29 \\
\hline 19 & 38.8 & 3.9 & 49 \\
\hline 63 & 40.6 & 12.9 & 155 \\
\hline 91 & 41.6 & 18.6 & 219 \\
\hline 106 & 34.3 & 21.7 & 309 \\
\hline 41 & 32.3 & 8.4 & 127 \\
\hline 38 & 23.2 & 7.8 & 164 \\
\hline 47 & 22.8 & 9.6 & 206 \\
\hline 16 & 11.8 & 3.3 & 135 \\
\hline 46 & 16.3 & 9.4 & 283 \\
\hline 489 & & & 1,695 \\
\hline
\end{tabular}

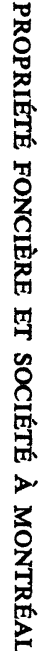

NOTE: Les individus propriétaires dans plusieurs quartiers ont été comptés autant de fois que leur nom apparaît dans un quartier.

SOURCE: Fonds Viger-Verreau 46, no 9, Archives du Séminaire de Québec. 
Le revenu annuel moyen pour l'ensemble de la cité s'établit ainsi: Canadiens, 50 livres; Anglo-Canadiens, 127 livres; Anglais et étrangers, 86 livres. Une simple moyenne ne rend pas compte de la complexité de la situation. La répartition des propriétaires selon des catégories de revenu permet de raffiner l'analyse. Le tableau 2 montre que la petite propriété est fortement concentrée entre les mains des Canadiens français qui, dans les catégories de moins de 50 livres, sont nettement sur-représentés. Dans les catégories de revenus élevés le partage est différent. Les Canadiens français sont sous-représentés mais, sauf une exception par ailleurs peu significative, ils rassemblent au moins $46 \%$ des effectifs de chaque catégorie. Les Anglais et étrangers concentrent leur présence dans ces catégories supérieures et y occupent une place plus grande que ne leur accorderait leur seul pourcentage de la population totale. Quant aux Anglo-Canadiens, s'ils se retrouvent plutôt dans les niveaux de revenus élevés ils y sont généralement sous-représentés.

Ce tableau n'est cependant que l'addition, au niveau de l'ensemble de la cité, des effectifs de chaque catégorie dans chacune des divisions de la cité. On n'y a pas éliminé les comptes doubles, c'est-à-dire les individus propriétaires dans plus d'un quartier. Les données de Viger permettent d'éliminer cette distorsion pour les catégories de revenus évalués à 100 livres et plus. C'est d'ailleurs dans ces catégories que risquent de se retrouver les propriétaires qui, dépassant le désir d'avoir une maison à eux, font véritablement de l'investissement foncier. Le tableau 3 nous présente une répartition d'où ont été éliminés les comptes doubles en regroupant toutes les propriétés d'un même individu.

Ces quelques données sur la composition ethnique du groupe des propriétaires fournissent des conclusions intéressantes. Les anglophones, qu'ils soient nés au Canada ou à l'étranger, obtiennent une majorité chez les grands propriétaires. Il y a là un phénomène de sur-représentation par rapport au pourcentage (45\%) des effectifs de la population totale. Qui s'en étonnerait quand on connaît le poids considérable de la bourgeoisie anglophone dans l'activité économique montréalaise à cette époque. $\mathrm{Au}$ surplus la distinction au'a établie Viger entre les Anglo-Canadiens et les autres est intéressante. Les personnes nées à l'étranger occupent au total une place importante, signe tangible de l'apport dynamique des immigrants à l'activité économique.

Le cas des propriétaires canadiens-francais est particulièrement intéressant. Nous avons vu qu'ils obtiennent 52.1\% du 
revenu foncier total de la cité. Bien sûr, la majorité des effectifs se concentre dans les propriétés à bas revenu, indice du désir des natifs du pays d'avoir une maison bien à eux, d'acheter un lot pour y vivre plutôt que pour $\mathrm{y}$ investir. Par contre on relève aussi une forte présence canadienne-française chez les propriétaires ayant un revenu annuel évalué à 100 livres et plus. Avec 46.9\% des effectifs à ce niveau les Canadiens français sont, à première vue, sous-représentés. Mais si on considère que, d'après les compilations de Ouellet, 17 ils ne rassemblent à cette époque qu'environ $35 \%$ des effectifs des hommes d'affaires et $40 \%$ de ceux des professions, on pourrait bien se trouver en face d'un phénomène de sous-représentation qui serait en fait une surreprésentation. On peut y voir une propension de la bourgeoisie canadienne-française à privilégier les investissements dans le secteur foncier. Nous reviendrons sur cette question plus loin.

TABLEAU 3

Répartition des propriétaires ayant un revenu de plus de 100 Livres Cité de Montréal, 1825

\begin{tabular}{|c|c|c|c|c|c|c|c|}
\hline \multirow{2}{*}{$\begin{array}{l}\text { Montant de } \\
\text { l'évaluation du } \\
\text { revenu annuel } \\
\text { (livres) }\end{array}$} & \multicolumn{2}{|c|}{$\begin{array}{c}\text { Canadiens } \\
\text { français }\end{array}$} & \multicolumn{2}{|c|}{$\begin{array}{l}\text { Anglo- } \\
\text { Canadiens }\end{array}$} & \multicolumn{2}{|c|}{$\begin{array}{l}\text { Anglais et } \\
\text { étrangers }\end{array}$} & \multirow{2}{*}{$\begin{array}{c}\text { Total } \\
\mathrm{n}\end{array}$} \\
\hline & $\mathrm{n}$ & & $\mathrm{n}$ & $\%$ & $\mathrm{n}$ & $\%$ & \\
\hline 600 et plus & 6 & 50.0 & 3 & 25.0 & 3 & 25.0 & 12 \\
\hline $500-599$ & 5 & 31.2 & 2 & 12.5 & 9 & 56.3 & 16 \\
\hline $400-499$ & 13 & 56.5 & 0 & 0 & 10 & 43.5 & 23 \\
\hline $300-399$ & 9 & 45.0 & 2 & 10.0 & 9 & 45.0 & 20 \\
\hline $200-299$ & 18 & 42.9 & 5 & 11.9 & 19 & 45.2 & 42 \\
\hline $100-199$ & 56 & 48.7 & 14 & 12.2 & 45 & 39.1 & 115 \\
\hline TOTAL & 107 & 46.9 & 26 & 11.4 & 95 & 41.7 & 228 \\
\hline
\end{tabular}

NOTE: Les individus propriétaires dans plusieurs quartiers ne sont comptés qu'une fois.

SOURCE: Fonds Viger-Verreau 46, no 9, Archives du Séminaire de Québec.

17 Fernand Ouellet, "Structure des occupations et ethnicité dans les villes de Québec et de Montréal (1819-1844)", Eléments d'histoire sociale du Bas-Canada (Montréal, HMH, 1972), 180-192. 
Avant de clore ce chapitre il faut dire quelques mots de ces grands propriétaires qui, bien que peu nombreux, accaparent une part appréciable du revenu foncier. Douze personnes ou institutions figurent au palmarès de la très grande propriété foncière montréalaise; il s'agit des propriétaires dont le revenu annuel est évalué entre 600 et 1,000 livres et plus: Pre Berthelet $(£ 2,129)$, D.-B. Viger $(£ 1,334)$, Sa Majesté $(£ 1,187)$, Austin Cuvillier (£907), J. Molson, père (£910), Le Séminaire (£912), David Ross (£880), Veuve Platt (£717), M. Lunn (£754), Succession David ( $£ 638)$, Bte Castonguay, père (£682), Félix Souligny $(£ 680)$.

Même s'il ne saurait être question d'étudier en détail ces très grands propriétaires dans le cadre de cette note, nous avons voulu tout de même voir un peu la composition et les caractéristiques de certains patrimoines canadiens-français. Nous avons retenu tous les "Canadiens" de la liste, soit P. Berthelet, D.-B. Viger, A. Cuvillier, B. Castonguay et Félix Souligny, auxquels nous avons ajouté Toussaint Pothier (578 livres). Ces propriétaires ont tous en commun un certain nombre de caractéristiques. La première est la diversité spatiale des propriétés; dans tous les cas, elles se trouvent au moins dans deux faubourgs, plus souvent dans trois. Ensuite ces ensembles sont ancrés dans la vieille ville où sont concentrées les plus hautes valeurs de propriété, à une exception près. Enfin tous les propriétaires résident dans la ville, sur l'un de leurs biens-fonds.

Prenons le cas de Berthelet. Le recensement fait par Viger indique que ses 23 propriétés, réparties dans trois faubourgs outre la ville, sont louées à 61 personnes. A l'intérieur de la ville, on relève 5 locations à des fins commerciales ou industrielles: une boutique où l'on fabrique des peignes, un coiffeur, une fabrique de chaises et deux fabriques de brosses et ouvrages de fil de fer. Dans le faubourg Saint-Laurent, la British and Canadian School utilise ses locaux. Notre propriétaire est partie prenante dans l'exploitation des immigrants entassés à la Pointeà-Callière, phénomène dont nous avons parlé plus haut. Il y possède trois propriétés dont l'une compte 43 résidents, chiffre élevé pour l'époque. La diversité du patrimoine de Berthelet nous le fait apparaître comme un véritable entrepreneur foncier. Il complète d'ailleurs ses activités dans un secteur connexe à l'habitation; possesseur de plusieurs poêles de fonte (environ 300 ), il mène un commerce lucratif de location, fournissant pour 
l'hiver ceux qui n'ont pas les moyens de s'acheter ce commode instrument. ${ }^{18}$

La situation de Denis-Benjamin Viger lui ressemble, le deuxième plus gros propriétaire loue également des locaux commerciaux, mais en plus, et, c'est là peut-être l'indice d'une future spéculation, il possède une terre avec grand jardin et verger dans le faubourg Saint-Louis. L'activité foncière d'un Cuvillier, d'un Castonguay et d'un Souligny est du même type.

Avec Toussaint Pothier, nous sommes déjà à un autre niveau: les locaux commerciaux sont absents, et on note un nombre important de lots - probablement non-bâtis - dans le patrimoine. Par contre il possède trois maisons avec jardin dans la ville.

Nous arrêtons ici ce rapide inventaire; il faudrait évidemment élargir l'éventail des cas étudiés et surtout analyser à fond l'origine et la composition de ces patrimoines fonciers pour tenter de dégager une typologie et les structures internes du phénomène. Toutefois ce premier regard sur le capital foncier montre toute la vigueur et l'importance de ce secteur dans la vie économique montréalaise.

Ces quelques constatations sur la propriété et les propriétaires à Montréal en 1825 ne sauraient épuiser le sujet. Elles n'en permettent pas moins d'apporter un éclairage nouveau à la connaissance de la société montréalaise du premier quart du $19 \mathrm{e}$ siècle. Elles ajoutent une autre dimension à notre perception des clivages sociaux et des clivages ethniques qui marquent cette société. Il faudra, dans une autre étape, affiner l'analyse, jumeler ces informations avec les données du recensement fait par Jacques Viger en 1825, recensement qui nous fournit les occupations, la taille des familles, le nombre de ménages par maison, etc. ${ }^{19} \mathrm{Il}$ faudra aussi compléter par le recours à d'autres sources.

Les conclusions qui se dégagent de cette étude sur 1825 sont doubles. On relève d'une part l'importance du capital foncier, ce qui montre déjà un début de concentration. Tout ce qui compte

18 Montréal fin de siècle: histoire de la métropole du Canada au dixneuvième siècle (Montréal, The Gazette Printing Company, 1899), 23.

19 Jean-Claude Robert et Claude Théorêt, "Le recencement de 1825", Groupe de recherche sur la société montréalaise au $19 \mathrm{e}$ siècle, Rapport 1972-1973 (Montréal, Université du Québec à Montréal, 1973), 15 p. (miméo). 
à Montréal dans le domaine du commerce et de l'industrie se retrouve au palmarès de la propriété et les sommes investies sont souvent considérables. D'autre part nous avons noté une assez forte participation canadienne-française, y compris dans les catégories supérieures de revenu foncier, participation qui surprend quand on connaît l'hégémonie des anglophones sur le grand commerce. Le comportement de Berthelet et des autres grands propriétaires canadiens-français ne semble pas laisser de doute sur le caractère capitaliste de leurs activités. Ces conclusions restent provisoires. Dégagées à partir de résultats globaux, elles devront être vérifiées par une analyse plus fine à la fois des sources utilisées ici et d'autres documents portant sur la période.

Ce travail ne serait guère plus qu'un exercice méthodologique s'il ne pouvait pas s'intégrer à une démarche scientifique cohérente et plus globale. A notre avis les données présentées ici permettent précisément de formuler une hypothèse plus générale à propos du rôle du capital foncier en milieu urbain et de la place qu'y occupe la bourgeoisie canadienne-française.

\section{Une hypothèse plus globale}

Profondément marqués par l'historiographie britannique et l'historiographie américaine, les historiens de l'économie canadienne ont valorisé l'entrepreneur et singulièrement le modèle anglais de l'entrepreneur, cet innovateur audacieux qui met sur pied l'industrie moderne. Alfred Dubuc a bien mis en lumière les déficiences que présente l'application de ce modèle au cas canadien. ${ }^{20}$

Dans un tel cadre interprétatif la bourgeoisie canadiennefrançaise fait figure de parent pauvre. Le problème qui retient l'attention est celui du petit nombre d'industriels canadiensfrançais et de la faible dimension de leurs entreprises. ${ }^{21}$ On oppose l'investissement industriel, qui serait un signe de dynamisme et de progrès, à l'investissement foncier ou immobilier qui serait un geste conservateur exprimant la recherche de

20 Alfred Dubuc, Thomas Molson, entrepreneur canadien, 1791-1863, Thèse de doctorat d'Etat (Paris, 1969) ; "Les classes sociales au Canada", Annales ESC, 22, 4 (juillet-août 1967): 829-844.

21 Voir René Durocher et Paul-André Linteau, Le "retard" du Québec et l'infériorité économique des Canadiens français (Montréal, Boréal Express, 1971), $127 \mathrm{p}$. 
sécurité et qu'on assimile au $20 \mathrm{e}$ siècle à l'achat d'obligations gouvernementales. L'article de Taylor illustre bien une telle interprétation. ${ }^{22}$

A notre avis cette présentation des faits, dominante dans l'historiographie canadienne, ne rend pas compte de la complexité de l'activité économique. A côté du capital financier, du capital industriel, du capital commercial il existe un capital foncier qui a une fonction spécialisée, celle d'administrer et d'aménager l'espace, sinon de le déterminer, facilitant ainsi les conditions de la production et de la circulation.

En milieu urbain on identifie peut-être trop ce type d'investissement à l'image de la spéculation foncière. Le phénomène nous paraît plus complexe. Si l'investisseur foncier spécule il va souvent plus loin en créant lui-même les conditions qui permettront la hausse de la valeur de ses terrains. Historiquement, en effet, les investisseurs dans le secteur foncier ont été de véritables promoteurs urbains. Ils font une exploitation capitaliste de leurs biens-fonds, construisent des édifices où ils entassent le plus grand nombre possible de locataires afin de maximiser leurs profits. Ils cherchent à concentrer la propriété afin de mieux en organiser le développement. Ils attirent des entreprises commerciales, industrielles, ferroviaires ou autres dont la présence augmentera la plus-value des terrains environnants. Ils manipulent ou à tout le moins exercent de fortes pressions sur les conseils municipaux afin que les décisions politiques aillent dans le sens de leurs intérêts économiques. Ils s'abouchent avec les dirigeants des entreprises de services publics (eau, gaz, tramways et électricité) dont la collaboration est essentielle à la réussite des projets de construction. Les historiens américains ont étudié le rôle déterminant de ces promoteurs dans la plupart des grandes villes des Etats-Unis. ${ }^{23}$

Il y a donc là une activité économique qui est évidemment liée aux progrès du commerce et de l'industrie mais dont il ne faudrait pas négliger l'impact.

Notre hypothèse est que la bourgeoisie canadienne-française, tout en participant de façon minoritaire au capital financier,

22 Norman W. Taylor, "L'industriel canadien-français et son milieu", R. Durocher et P.-A. Linteau, op. cit., 43-74.

23 Voir C. N. Glaab et A. T. Brown, A History of Urban America (Macmillan, 1967), 107-132; D. J. Boorstin, "The Businessman as an American Institution", A. B. Callow, ed., American Urban History (New York, Oxford L'niversity Press, 1969), 136-143. 
commercial ou industriel a eu véritablement ses assises économiques dans le secteur foncier. Que, loin d'y voir un placement nécessairement conservateur et exclusivement passif, elle a cherché à faire une exploitation capitaliste de ce patrimoine foncier qui s'accumulait de génération en génération.

Si cette hypothèse s'avérait juste elle résoudrait cette apparente contradiction entre le contrôle idéologique qu'a exercé cette bourgeoisie sur les masses canadiennes-françaises et la faiblesse de ses assises économiques dans le secteur industriel. Le capital foncier est, lui aussi, source de pouvoir et de domination. Dans un autre contexte, celui d'une économie agro-forestière, Normand Séguin a bien montré la véritable dépossession dont sont victimes les colons du Lac Saint-Jean à la fin du $19 \mathrm{e}$ siècle. ${ }^{24}$ On y assiste à un accaparement du sol par la bourgeoisie qui exerce ainsi un contrôle social poussé.

Bien sûr, les Canadiens français ne sont pas seuls à investir dans ce secteur, mais leur présence est là beaucoup plus forte qu'ailleurs. Ils y ont sans doute plus facilement accès aux réseaux d'information qui, dans le cas de l'industrie et du commerce, leur sont trop souvent fermés.

Cette hypothèse sur l'importance du capital foncier pour la bourgeoisie canadienne-française nous paraît étayée par quelques indices. Le cas de Montréal en 1825 que nous avons présenté plus haut est révélateur. Et que dire de l'activité d'un Barthélemy Joliette qui pour augmenter des revenus seigneuriaux qui plafonnent, monte une industrie $d u$ bois et fait du développement urbain dans son village d'Industrie..$^{25} \mathrm{~A}$ la fin du $19 \mathrm{e}$ siècle la question de la construction d'installations portuaires dans l'est de Montréal oppose en deux camps ennemis la bourgeoisie francophone et anglophone. ${ }^{26}$ Cette affaire illustre l'importance des investissements fonciers canadiens-français dans cette partie de la ville. Enfin l'étude du développement de la ville de Maison-

24 Normand Séguin, "Hébertville au Lac Saint-Jean (1850-1900), un exemple québécois de colonisation au XIXe siècle", Société historique du Canada, Communications historiques 1973, à paraître.

25 Jean-Claude Robert, "Un seigneur entrepreneur, Barthélemy Joliette et la fondation du village d'Industrie (Joliette), 1822-1850", RHAF, 26, 3 (décembre 1972) : 375-395; du même auteur, L'activité économique de Barthélemy Joliette et la fondation du village d'Industrie (Joliette) 1822-1850. Thèse de M. A. (histoire), (Université de Montréal, 1971), $183 \mathrm{p}$.

${ }^{26}$ Paul-André Linteau, "Le développement du port de Montréal au début du 20e siècle", Société historique du Canada, Communications historiques 1972 (Ottawa, 1974) : 181-185. 


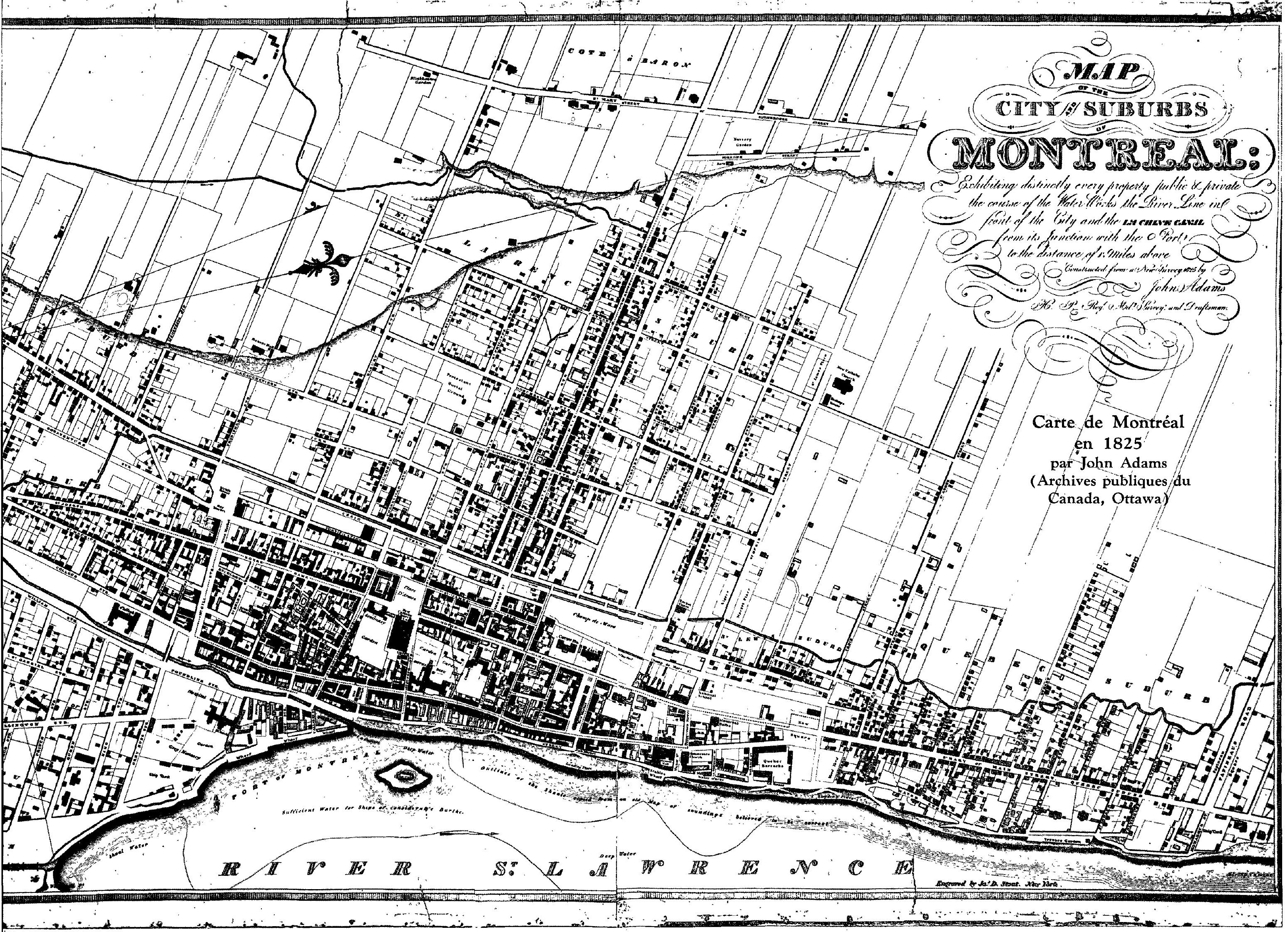


neuve, en banlieue de Montréal, nous a permis de voir à l'œuvre ces promoteurs canadiens-français qui, propriétaires de grands terrains, les mettent en valeur rapidement et avec profit en contrôlant le conseil municipal et en faisant adopter une politique industrielle favorisant le peuplement. ${ }^{27}$

Ces quelques indices sont encore insuffisants pour prouver une hypothèse. Il faudra des recherches plus nombreuses et plus poussées. Les travaux en cours permettent toutefois d'espérer que nous aurons bientôt une meilleure compréhension du rôle du capital foncier dans l'économie québécoise.

27 Paul-André Linteau, Histoire de la ville de Maisonneuve, 1883-1918, thèse de $\mathrm{Ph}$. $\mathrm{D}$. en préparation. 